Case Report

\title{
RADIO ULNAR SYNOSTOSIS: A CASE REPORT
}

\author{
Ramakrishna Avadhani ${ }^{1}$, Bindhu S. ${ }^{1}$, Vikram ${ }^{1}$, Dhanesh Kumar K.U. ${ }^{2}$ \\ \& Arunachalam Kumar ${ }^{3}$ \\ ${ }^{1}$ Department of Anatomy, Yenepoya M edical College, Yenepoya University, M angalore - 575018 , \\ ${ }^{2}$ Nitte Institute of Physiotherapy, Nitte University, M angalore - 575018 \\ ${ }^{3}$ Department of Anatomy, K. S Hegde M edical Academy, Nitte University, M angalore - 575018 \\ Correspondence: \\ E-mail : dean.keshema@nitte.edu.in Tel: +91 824 2204490, Fax: +91 8242204162
}

\begin{abstract}
:
Congenital radio-ulnar synostosis is caused by a failure of segmentation between the radius and ulna. It occurs rarely, with approximately 350 cases reported. Congenital radioulnar synostosis is equally affected in males and females with half of patients showing bilateral involvement. While most cases of congenital radio-ulnar synostosis occur spontaneously around one in five cases is associated with a family history of the same affliction. This condition can also occur as part of an underlying syndrome or in association with other developmental abnormalities. Congenital radio ulnar synostosis is diagnosed by clinicians through medical history and careful physical examination. X-rays or CT scans are be used to confirm the deformity. The diagnosis of congenital radio-ulnar synostosis is more often made in late childhood or at adolescence, as pain is rarely a complaint and much mobility of elbow flexion and extension preserved. In relatively mild cases, when patients themselves may notice subtle limitations or differences in forearm rotation the diagnosisistypically made at an earlier age.
\end{abstract}

Keywords: Synostosis; interosseus membrane; ossification; segmentation

\section{Introduction:}

Synostosis is the union of any adjacent bones, can involve any part of the body. Synostosis between the radius and ulna can take 2 forms, congenital and post traumatic. The degree of fusion in radio-ulnar synostosis varies and may not involve the radial head (1). The initial description of congenital radio-ulnar synostosis was provided by Sandifort in 1793.

The condition is caused by a failure of segmentation between the radius and ulna. Embryologically, the upper limb bud arises from the unsegmented body wall at 25-28 days. The elbow becomes visible at 34 days and the humerus, radius and ulna become visible at 37 days. Initially, the 3 cartilagenous analogs of these three bones connected before segmentation; therefore, for a transient phase, the radius and ulna share a common perichondrium. Abnormal events during this period could lead to a failure of segmentation. The duration and severity of the insult determines the degree of subsequent synostosis. Endochondral ossification then proceeds and the cartilaginous synostosis ossifies either partially or completely, in the longitudinal or transverse plane (2). In the forearm, congenital radio-ulnar synostosis usually occurs between the proximal radius and ulna. Although the condition is present at birth, it is usually not discovered until early adolescence, when the patient presents with a lack of pronation and supination. Initially the union may be more of a synchondrosis, but as the skeleton matures or the osseuos bridge between the radius and ulna becomes more radiographically apparent.

The exact incidence of this condition is unknown. Males and females are equally affected and over half patients having bilateral involvement. Congenital radio-ulnar synostosis is classified into type 1 and type 2.In type 1 , complete synostosis occurs, with radius and ulna fused proximally for variable distance. Type 2 shows less involvement and may exist as a partial union. Type 2 involves the region just distal to the proximal radial epiphysis and is associated with radial head dislocation (3). Congenital synostosis can be further classified into several categories, fibrous synostosis, bony synostosis, associated posterior dislocation of the radius or associated anterior dislocation of the radius (4). Congenital synostosis can display a spectrum of anomalies in with fusion occurring in varying lengths and with or without involvement of the radial head (5).

\section{Case report :}

The patient was a 17 year old boy had limited range of motion of both the forearms. The patient had right-hand 
dominance and denied any weakness or loss of feeling in his forearms. Some of the movements, especially supination was more difficult than others. The boy has no significant past history and had had a normal developmental history. His sister also has unilateral radioulnar synostosis (in left forearm). He did not have any history of any trauma or fractures. The forearms do not cause him functional problems and he suffers no pain.

On physical examination the right elbow hand flexion from 0 to $145^{\circ}$, shoulder flexion from 0 to $180^{\circ}$ but the forearm was fixed with limited / restricted supination and had pronation from 0 to $10^{\circ}$ associated with accessory movements activated from shoulder. The wrist had an ulnar deviation from $0-40^{\circ}$, radial deviation from 0 to $20^{\circ}$, extension from 0 to $70^{\circ}$, flexion from 0 to $50^{\circ}$. Thumb

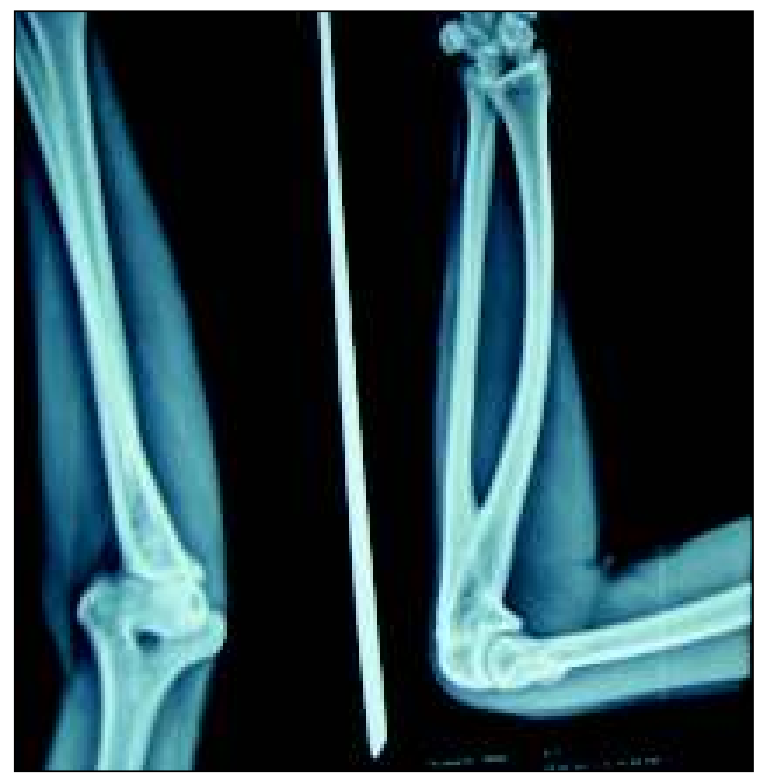

AP and lateral views of left forearm: showing fusion of upper end of radius with ulna

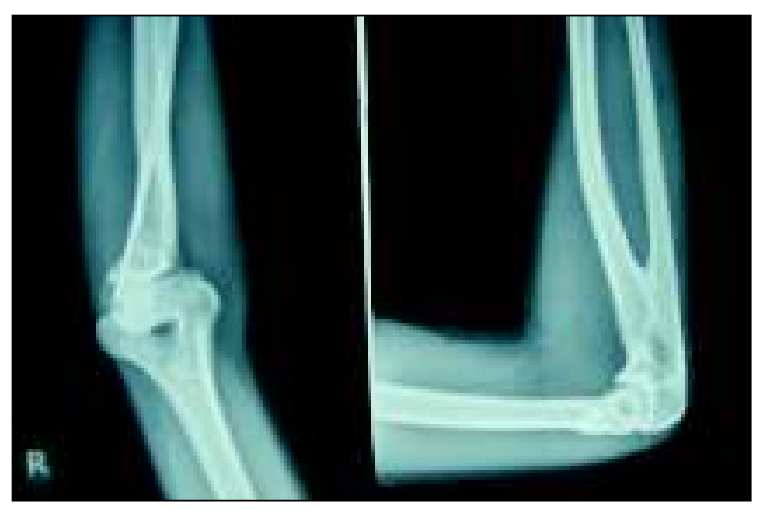

AP and lateral views of right forearm: showing fusion of upper end of radius with ulna

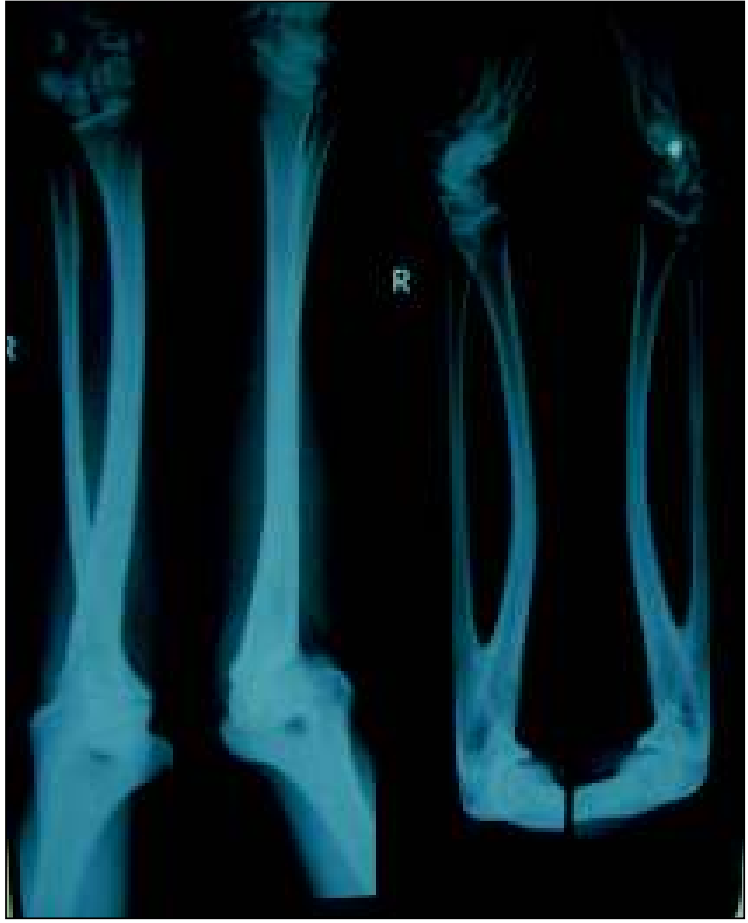

AP and lateral views of both forearms: showing fusion of upper end of radius with ulna

movements are normal with muscle power grade 5/5. Left elbow had flexion from 0 to $145^{\circ}$, shoulder flexion from 0 to $180^{\circ}$ but forearm is fixed with restricted supination, pronation associated with accessory movements activated from shoulder. The wrist had an ulnar deviation from 0 to $45^{\circ}$ and radial deviation from 0 to $15^{\circ}$, extension, 0 to $70^{\circ}$ Left thumb movements were normal. Left hand grip function was slightly compromised compared to the right and presented a mild grade of kinetic tremor on voluntary action. M uscle power grade was $5 / 5$.

Radiographs of both the arms demonstrate a bony synostosis of proximal radius and ulna and spectrum of anatomic variation, absence of radial head, forearm shortening and radial shaft bowing. The patient does not have the complaint of hyper mobility of wrist or difficulty in holding or use small objects.

\section{Discussion :}

Congenital radio-ulnar synostosis is caused by an in-utero insult with recorded reports of its association with general skeletal abnormalities such as hip dislocation, knee anomalies, clubfoot, polydactyly, syndactyly, Madelung deformity, ligamentous laxity, thumb hypoplasia, carpal 
coalition and problems of cardiac, renal, neurologic and GI systems. Some associated abnormalities and syndromes are genetically determined, including acrocephalicsyndactyly, Apert syndrome, Carpenter syndrome and Fetal Alcohol syndrome (6).

The skeletal anomaly includes varying degrees of proximal fusion of radial and ulnar, with or without involvement of radial head. If the radial head is involved, it may be dislocated anteriorly or posteriorly (7). Functional deficits associated with congenital radio-ulnar synostosis depend on the severity of the deformity and on whether or not it is bilateral. Hypermobility at the midcarpal and radiocarpal joints can disguise the lack of forearm rotation, particularly with neutral or mild pronation deformities. There is usually full or nearly full elbow range of motion, with flexion contractures rarely exceeding $30^{\circ}$. An abnormal 'carrying angle' of the elbow or a shortening of the forearm may be observed. Pain is not a presenting symptom until teenage

\section{References:}

1. Hansen $\mathrm{OH}$, Anderson NO. Congenital radioulnar synostosis. Report of 37 cases. Acta Orthop Scand.1970; 41(3):225-30.

2. Lewis WH. The development of the arm in man. Am J Anat 1901;1:145-83

3 Wilkie DP. Congenital radioulnar synostosis. Br J Surg.1914;1:366-75

4 ClearyJE, Omer GE.Congenital proximal radioulnar synostosis. Natural history and functional assessment. J Bone joint Surg Am. Apr 1985;67(4):539-45

5 Simmons BP, Southmayd WW, Riseborough EJ. Congenital radioulnar years, when progressive and symptomatic radial head subluxation may be noted. The disability is most significant in bilateral cases with severe problems in pronation. Indications for surgical interventions in congenital radioulnar synostosis still remain somewhat controversial but are related to bilaterality and to the degree of deformity (8). Wrist hypermobilty allows further functional compensation but in our patient wrist mobility was normal. Severe pronation deformities cause significant functional difficulty, especially with activities requiring supination.

Surgery should be performed in childhood before patients are school-aged. The rationale for surgery is dictated more on the individual functional limitations rather than on absolute forearm position. Surgery can be performed either to remove the abnormal soft tissue or bony connection or to re-position the forearm to a better functionally acceptable position (9).

synostosis. J Hand Surg Nov 1983; 8(6):829-38

6 Jaffer Z,Nelson M, BeightonP. Bone fusion in the foetal alcohol syndrome.J Bone Joint Surg Br.1981; 63 B (4):569-71.

7 Mital MA. Congenital radioulnar synostosis and congenital dislocation of the radial head. Orthop Clin North A. Apr 1976; (2):375-83.

8 Dawson HG.,A Congenital deformity of the forearm and its operative treatment.Br M edJ.1912:833-5

9 Khalil Vizkeley T. Osteotomy of the synostosis mass for the treatment of congenital radioulnar synostosis. Arch Orthop Trauma Surg. 1993;113(1):20-2 